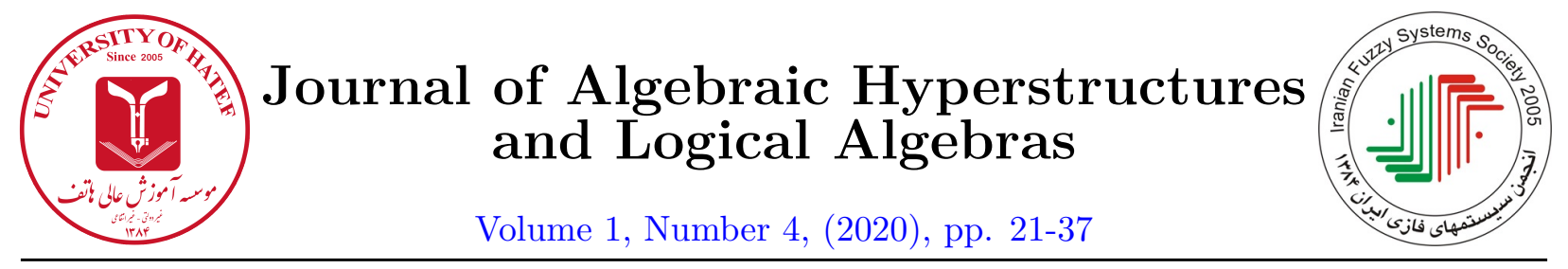

\title{
Some types of derivations in bounded commutative residuated lattices
}

\author{
D.L. Keubeng Yemene ${ }^{1}$, L.E. Diekouam Fotso ${ }^{2}$, D. Akume ${ }^{3}$ and C. Lele \\ 1,4 Department of Mathematics and Computer Science, University of Dschang, Dschang, Cameroon \\ ${ }^{2}$ Department of Mathematics, HTTC Maroua University of Maroua, Maroua, Cameroon \\ ${ }^{3}$ Computer Science Department, HTTTC Kumba University of Buea, Cameroon
}

darlinekeubeng@yahoo.fr, lucdiekouam@yahoo.fr,d-akume@yahoo.ca, celestinlele@yahoo.com

\begin{abstract}
In this paper, the notion of mutiplicative derivation, pseudo implicative derivation and implicative derivation on a bounded commutative residuated lattice are presented with some useful examples. We generalized these notions of derivation by introducing $(f, g)$-multiplicative derivation, $(f, g)$-pseudo implicative derivation and $(f, g)$-implicative derivation, and discussed some related properties; the conditions for $(f, g)$-multiplicative derivation, $(f, g)$-pseudo implicative derivation and $(f, g)$-implicative derivation to be monotone are provided. The set of fixed points is defined by using the notion of $(f, g)$-multiplicative derivation of bounded commutative residuated lattices. We also analyzed the link between different types of derivation.
\end{abstract}

\section{Article Information}

Corresponding Author:

L.E. Diekouam Fotso;

Received: June 2020;

Revised: August 2020;

Accepted: August 2020,

Paper type: Original.

Keywords:

Bounded commutative residuated lattice, Boolean algebra, derivation, set of fixed points, $(f, g)$-derivation.

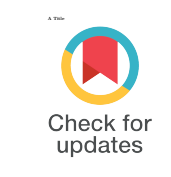

\section{Introduction}

The various logical algebras have been proposed as the semantical systems of non-classical logic. Among these logical algebras, bounded commutative residuated lattices are very important algebraic structures. Residuated lattices constitute the semantics of Höhle's monoidal logic [7, 9], which are the basis for the majority of formal fuzzy logics.

The notion of derivation, which comes from mathematical analysis, is also useful to study some structural properties of various kinds of algebra, in particular it made it possible to characterize distribitive and modular lattices.

https://doi.org/10.29252/HATEF.JAHLA.1.4.2 
The concept of derivation has been introduced in the commutative rings by Posner in [10], in $B C I$ algebra by Y. B. Jun and X. L. Xin in 2004[8], in lattice ([12],[5]), in $M V$-algebra [2], $B L$-algebra [1] and recently in residuated lattice [6]. In this paper, we generalized the notion of derivation on residuated lattice, by introducing the notion of $(f, g)$-derivation on bounded commutative residuated lattice and studying some important properties.

This paper is organized as follows: in Section 2, we review some basic notions and properties of bounded commutative residuated lattices; in Section 3, we introduce the notion of multiplicative derivation, pseudo implicative derivation and implicative derivation of bounded commutative residuated lattices with some useful examples. As extension of these notions of derivation, we introduce in Section 4, the notions of $(f, g)$-multiplicative derivation, $(f, g)$-pseudo implicative derivation and $(f, g)$-implicative derivation and investigate some properties of them.

\section{Preliminaries}

In this section, we review some basic definitions on bounded commutative residuated lattices.

Definition 2.1. [9], [13] A bounded commutative residuated lattice is an algebra $(L, \wedge, \vee, \odot, \rightarrow, 0,1)$ of type $(2,2,2,2,0,0)$ such that for all $x, y, z \in L$,

$\left(L_{1}\right)(L, \wedge, \vee, 0,1)$ is a bounded lattice,

$\left(L_{2}\right)(L, \odot, 1)$ is a commutative monoid,

$\left(L_{3}\right) x \odot y \leq z$ if and only if $y \leq x \rightarrow z$.

- Let $L$ be a bounded commutative residuated lattice, for any $x \in L$, we define $x^{*}:=x \rightarrow 0$. According to [6], we adopt the notations $x \oslash y=x^{*} \rightarrow y$ for every $x, y \in L,\left(x^{*}\right)^{*}=x^{* *}, x^{0}=1$ and $x^{n}=x^{n-1} \odot x$ for natural number $n \geq 1$.

- Let $L$ be a bounded commutative residuated lattice. According to [14], we define the pseudo implication operation $\rightarrow$ by $x \rightarrow y=x \odot y^{*}$ for any $x, y \in L$.

We will denote by the letter $L$ the 7 -tuple $(L, \wedge, \vee, \odot, \rightarrow, 0,1)$.

Definition 2.2. [11] A bounded commutative residuated lattice $L$ is called BL-algebra, if the following two identities hold in L:

(1) $x \odot(x \rightarrow y)=x \wedge y$,

(divisibility),

(2) $(x \rightarrow y) \vee(y \rightarrow x)=1$,

(prelinearity).

Definition 2.3. [9] A bounded commutative residuated lattice $L$ is regular if for all $x \in L, x^{* *}=x$.

Definition 2.4. [4] A bounded commutative residuated lattice $L$ is called a G-algebra (Gödel algebra) if it is a BL-algebra such that for all $x \in L, x \odot x=x$.

Definition 2.5. [9] An ideal of a bounded commutative residuated lattice $L$ is a non-empty subset $I$ of $L$ that for any $x, y \in L$, satisfying in the following conditions:

(1) $x, y \in I$ implies $x \oslash y \in I$,

(2) if $x \leq y$ and $y \in I$, then $x \in I$.

Theorem 2.6. [9] Let $L$ be a bounded commutative residuated lattice. Then $I$ is an ideal of $L$ if and only if I satisfies the following conditions: 
(1) $0 \in I$,

(2) for any $x, y \in L$, if $x \odot y^{*} \in I$ and $x \in I$, then $y \in I$.

Definition 2.7. [6] Let $S$ and $T$ be two posets. We shall say that a pair $(d, g)$ of functions $d: S \rightarrow T$ and $g: T \rightarrow S$, is a Galois connection or an adjunction between $S$ and $T$ if $d$ and $g$ satisfy:

(1) $d$ and $g$ are monotone,

(2) $d(s) \leq t$ if and only if $s \leq g(t)$ for all $s \in S$ and $t \in T$.

Now, we give some properties of the bounded commutative residuated lattices necessary in the proofs of some results.

Proposition 2.8. [9] Let $L$ be a bounded commutative residuated lattice, for any $x, y, z \in L$, we have the following properties:

$\left(L_{4}\right) 1 \rightarrow x=x, x \rightarrow x=1,1^{*}=0,0^{*}=1, x \rightarrow 1=1,0 \rightarrow x=1$,

$\left(L_{5}\right) x \odot y \leq x, y, x \odot y \leq x \wedge y$ and $x \odot 0=0$,

$\left(L_{6}\right) x \odot y \leq x \rightarrow y$ and $y \leq x \rightarrow y$,

$\left(L_{7}\right) x \leq y$ if and only if $x \rightarrow y=1$,

$\left(L_{8}\right) x \rightarrow y=y \rightarrow x=1$ if and only if $x=y$,

$\left(L_{9}\right) x \odot(x \rightarrow y) \leq x$ and $y \leq(x \rightarrow y) \rightarrow y$,

$\left(L_{10}\right) x \odot(y \rightarrow z) \leq y \rightarrow(x \odot z) \leq(x \odot y) \rightarrow(x \odot z)$,

$\left(L_{11}\right) x \rightarrow y \leq(x \odot z) \rightarrow(y \odot z)$,

$\left(L_{12}\right) x \leq y$ implies $x \odot z \leq y \odot z$,

$\left(L_{13}\right) x \leq y$ implies $z \rightarrow x \leq z \rightarrow y$ and $y \rightarrow z \leq x \rightarrow z$

$\left(L_{14}\right) x \odot(y \vee z)=(x \odot y) \vee(x \odot z)$ and $x \odot(y \wedge z)=(x \odot y) \wedge(x \odot z)$,

$\left(L_{15}\right) x \rightarrow(y \rightarrow z)=y \rightarrow(x \rightarrow z)$.

Theorem 2.9. [4, 9] Let $L$ be a bounded commutative residuated lattice, if $x, y, z \in L$, then we have

$\left(L_{16}\right) x \odot x^{*}=0$ and $x \odot y=0$ if and only if $x \leq y^{*}$,

$\left(L_{17}\right) x \leq x^{* *}, x^{* *} \leq x^{*} \rightarrow x$ and $x^{* * *}=x^{*}$,

$\left(L_{18}\right) x \rightarrow y \leq y^{*} \rightarrow x^{*}$ and $(x \odot y)^{*}=x \rightarrow y^{*}=y \rightarrow x^{*}=x^{* *} \rightarrow y^{*}$,

$\left(L_{19}\right) x \leq y$ implies $y^{*} \leq x^{*}$,

$\left(L_{20}\right) x \rightarrow(y \wedge z)=(x \rightarrow y) \wedge(x \rightarrow z)$. 
Every residuated lattice satisfying $x \vee x^{*}=1$ is called a Boolean algebra. We recall that for a bounded lattice $L$, it is common to denote by $B(L)$ the Boolean lattice of complemented elements in $L$.

Theorem 2.10. [1] Let $L$ be a bounded commutative residuated lattice, for $x \in L$, the following statements are equivalent if $L$ is a BL-algebra:

(1) $x \in B(L)$,

(2) $x \odot x=x$ and $x^{* *}=x$,

(3) $x^{*} \rightarrow x=x$,

(4) $x^{*} \vee x=1$,

(5) $(x \rightarrow e) \rightarrow x=x$, for any $e \in L$.

More generaly we have:

$(1) \Rightarrow(2),(3),(4)$ and (5). If $e \in B(L)$, then $e \odot x=e \wedge x$, for all $x \in L$.

Definition 2.11. [13] Let $L$ and $L^{\prime}$ be two bounded commutative residuated lattices, $f: L \rightarrow L^{\prime}$ be a homomorphism of bounded commutative residuated lattices if for all $x, y \in L$ :

(i) $f(x \wedge y)=f(x) \wedge f(y)$ and $f(x \vee y)=f(x) \vee f(y)$,

(ii) $f(x \odot y)=f(x) \odot f(y)$,

(iii) $f(x \rightarrow y)=f(x) \rightarrow f(y)$,

(iv) $f(0)=0$.

The following lemma (where we have added the straightforward results (2) and (6)) and theorem stated in the case of BL-algebra [1, 14] are true in bounded commutative residuated lattice.

Lemma 2.12. 14] Let $L$ be a BL-algebra, for any $x, y, z \in L$, we have:

(1) $x \rightarrow 0=x, 0 \rightarrow x=0$ and $x \rightarrow x=0$,

(2) $x \rightarrow 1=0$ and $1 \rightarrow x=x^{*}$,

(3) $x \rightarrow y \leq x$, if $x \leq y$, then $x \rightarrow y=0$,

(4) $x \leq y$ implies $z \rightarrow y \leq z \rightarrow x$ and $x \rightarrow z \leq y \rightarrow z$,

(5) $x \rightarrow y=0$ implies $y^{*} \leq x^{*}$.

(6) If $x \in B(L)$, then $1 \rightarrow x^{*}=x$.

Theorem 2.13. [1] Let $L, L^{\prime}$ be two BL-algebras and $f: L \rightarrow L^{\prime}$ be a homomorphism. For all $x, y \in L$, we have:

(1) $f\left(x^{*}\right)=(f(x))^{*}, f(1)=1$,

(2) If $x \leq y$, then $f(x) \leq f(y)$,

(3) $f(x \oslash y)=f(x) \oslash f(y)$. 


\section{Derivations of bounded commutative residuated lattices}

In the following section, we extend some results from [1] in the context of bounded commutative residuated lattice with some useful examples. We will see that the identity function is a mutiplicative derivation and implicative derivation, but it is pseudo implicative derivation only if $L$ is a G-algebra.

Definition 3.1. Let $L$ be a bounded commutative residuated lattice, then a map $d: L \rightarrow L$ is called:

(1) A mutiplicative derivation, if $d(x \odot y)=(d(x) \odot y) \vee(x \odot d(y))$, for all $x, y \in L$,

(2) A pseudo implicative derivation, if $d(x \rightarrow y)=(d(x) \rightarrow y) \odot(x \rightarrow d(y))$, for all $x, y \in L$,

(3) An implicative derivation, if $d(x \rightarrow y)=(d(x) \rightarrow y) \vee(x \rightarrow d(y))$, for all $x, y \in L$.

For a residuated lattice $L$, we will denote by $d_{m}$ the multiplicative derivation, $d_{p}$ the pseudo implicative derivation and $d_{i}$ the implicative derivation.

Example 3.2. Let $L=\{0, a, b, c, 1\}$ such that the order on $L$ is given by the following Hasse diagram:

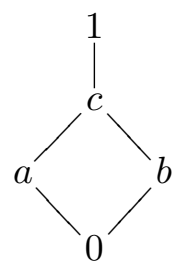

The operations $\odot$ and $\rightarrow$ on $L$ are defined by the following tables:

\begin{tabular}{c|ccccc}
$\rightarrow$ & 0 & $\mathrm{a}$ & $\mathrm{b}$ & $\mathrm{c}$ & 1 \\
\hline 0 & 1 & 1 & 1 & 1 & 1 \\
$\mathrm{a}$ & $\mathrm{b}$ & 1 & $\mathrm{~b}$ & 1 & 1 \\
$\mathrm{~b}$ & $\mathrm{a}$ & $\mathrm{a}$ & 1 & 1 & 1 \\
$\mathrm{c}$ & 0 & $\mathrm{a}$ & $\mathrm{b}$ & 1 & 1 \\
1 & 0 & $\mathrm{a}$ & $\mathrm{b}$ & $\mathrm{c}$ & 1
\end{tabular}

\begin{tabular}{c|ccccc}
$\odot$ & 0 & $\mathrm{a}$ & $\mathrm{b}$ & $\mathrm{c}$ & 1 \\
\hline 0 & 0 & 0 & 0 & 0 & 0 \\
$\mathrm{a}$ & 0 & $\mathrm{a}$ & 0 & $\mathrm{a}$ & $\mathrm{a}$ \\
$\mathrm{b}$ & 0 & 0 & $\mathrm{~b}$ & $\mathrm{~b}$ & $\mathrm{~b}$ \\
$\mathrm{c}$ & 0 & $\mathrm{a}$ & $\mathrm{b}$ & $\mathrm{c}$ & $\mathrm{c}$ \\
1 & 0 & $\mathrm{a}$ & $\mathrm{b}$ & $\mathrm{c}$ & 1
\end{tabular}

Then $(L, \wedge, \vee, \odot, \rightarrow, 0,1)$ is a residuated lattice which is not a BL-algebra because

$$
(a \rightarrow b) \vee(b \rightarrow a)=b \vee a=c \neq .
$$

We have some multiplicative derivations on $L$ is defined by $d_{m, 0}(x)=0$ for all $x \in L$ and $d_{m, 1}=$ $i d_{L}$.

$$
d_{m, 2}(x)=\left\{\begin{array}{lll}
0 & \text { if } & x \in\{0, a, c, 1\} \\
b & \text { if } & x=b
\end{array} \quad d_{m, 3}(x)=\left\{\begin{array}{lll}
0 & \text { if } & x \in\{0, a, 1\} \\
b & \text { if } & x \in\{b, c\}
\end{array}\right.\right.
$$

Some pseudo implicative derivations on $L$ are defined by $d_{p, 1}=i d_{L}$ and $d_{p, 0}(x)=0, \forall x \in L$.

$$
d_{p, 2}(x)=\left\{\begin{array}{lll}
0 & \text { if } & x \in\{0, b, c, 1\} \\
a & \text { if } & x=a
\end{array} \quad d_{p, 3}(x)=\left\{\begin{array}{lll}
0 & \text { if } & x \in\{0,1\} \\
a & \text { if } & x \in\{a, c\} \\
b & \text { if } & x=b
\end{array}\right.\right.
$$


Some implicative derivations on $L$ are $d_{i, 0}(x)=1, \forall x \in L ; d_{i, 1}=i d_{L}$;

$$
d_{i, 2}(x)=\left\{\begin{array}{lll}
a & \text { if } & x=0 \\
1 & \text { if } & x \neq 0
\end{array} \quad d_{i, 3}(x)=\left\{\begin{array}{lll}
1 & \text { if } & x \in\{1, c, b\} \\
a & \text { if } & x=a \\
b & \text { if } & x=0
\end{array}\right.\right.
$$

Proposition 3.3. Let $L$ be a bounded commutative residuated lattice and $d_{m}$ be a mulplicative derivation on $L$. Then the following statements hold:

(1) If $x \leq y$, then $d_{m}(x) \leq y^{* *}$ and $d_{m}\left(y^{*}\right) \leq x^{*}$. Moreover, $y \in B(L)$ implies $d_{m}(x) \leq y$,

(2) $d_{m}(x) \leq x^{* *}$, and moreover $x \in B(L)$ implies $d_{m}(x) \leq x$.

Proof. (1) Since $x \leq y$, by Theorem 2.9 we have $x \odot y^{*}=0$. Therefore, $0=d_{m}(0)=\left(d_{m}(x) \odot y^{*}\right) \vee$ $\left(x \odot d_{m}\left(y^{*}\right)\right)$. That is $d_{m}(x) \odot y^{*}=0$ and $x \odot d_{m}\left(y^{*}\right)=0$; hence $d_{m}(x) \leq y^{* *}$ and $d_{m}\left(y^{*}\right) \leq x^{*}$ by Theorem 2.9 and Proposition 2.8. Since $y \in B(L)$, we have $y^{* *}=y$, and so $d_{m}(x) \leq y$.

(2) By taking $x=y$ in (1) we obtain the result.

Proposition 3.4. Let $L$ be a bounded commutative residuated lattice and $d_{p}$ be a pseudo implicative derivation on $L$. Then the following statements hold:

(1) $d_{p}(0)=0$,

(2) $d_{p}(x) \leq x$, for all $x \in L$.

Proof. (1) Since $d_{p}(0)=d_{p}(x \rightarrow 1)$ for all $x \in L$, we get $d_{p}(0)=\left(d_{p}(x) \rightarrow 1\right) \odot\left(x \rightarrow d_{p}(1)\right)=0$, hence $d_{p}(0)=0$.

$(2) d_{p}(x)=d_{p}(x \rightarrow 0)=\left(d_{p}(x) \rightarrow 0\right) \vee\left(x \rightarrow d_{p}(0)\right)=\left(d_{p}(x) \odot 1\right) \vee(x \odot 1)=d_{p}(x) \vee x$, hence $d_{p}(x) \leq x$.

Theorem 3.5. [6] Let $L$ be a bounded commutative residuated lattice and $d_{i}$ be an implicative derivation on $L$, then for all $x, y \in L$, we have:

(1) $d_{i}(1)=1$,

(2) $d_{i}(x) \geq x$,

(3) $d_{i}(x) \vee d_{i}(y)=d_{i}(x \rightarrow y)$,

(4) $d_{i}(x \wedge y)=d_{i}(x) \wedge d_{i}(y)$.

Remark 3.6. Let $i d_{L}$ be the identity function on $L$, it is easy to see that $i d_{L}$ is a multiplicative derivation and an implicative derivation on $L$. If $L$ is a $G$-algebra, then $i d_{L}$ is a pseudo implicative derivation on $L$. The following example show that the converse is not always true.

Example 3.7. Let $L=\{0, a, b, c, d, 1\}$ be a set with Hasse diagram and Cayley tables as follows:

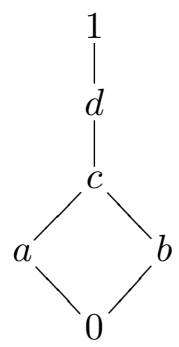

The operations $\odot$ and $\rightarrow$ on $L$ are defined by the following tables: 


\begin{tabular}{c|cccccc}
$\rightarrow$ & 0 & $a$ & $b$ & $c$ & $d$ & 1 \\
\hline 0 & 1 & 1 & 1 & 1 & 1 & 1 \\
$a$ & $b$ & 1 & $b$ & 1 & 1 & 1 \\
$b$ & $a$ & $a$ & 1 & 1 & 1 & 1 \\
$c$ & 0 & $a$ & $b$ & 1 & 1 & 1 \\
$d$ & 0 & $a$ & $b$ & $d$ & 1 & 1 \\
1 & 0 & $a$ & $b$ & $c$ & $d$ & 1
\end{tabular}

\begin{tabular}{c|cccccc}
$\odot$ & 0 & $a$ & $b$ & $c$ & $d$ & 1 \\
\hline 0 & 0 & 0 & 0 & 0 & 0 & 0 \\
$a$ & 0 & $a$ & 0 & $a$ & $a$ & $a$ \\
$b$ & 0 & 0 & $b$ & $b$ & $b$ & $b$ \\
$c$ & 0 & $a$ & $b$ & $c$ & $c$ & $c$ \\
$d$ & 0 & $a$ & $b$ & $c$ & $c$ & $d$ \\
1 & 0 & $a$ & $b$ & $c$ & $d$ & 1
\end{tabular}

Then $(L, \wedge, \vee, \odot, \rightarrow, 0,1)$ is a residuated lattice which is not a BL-algebra because $(a \rightarrow b) \vee(b \rightarrow$ $a)=b \vee a=c \neq 1$; and is not a G-algebra because $d \odot d=c$. Since $i d_{L}(d \rightarrow 0)=i d_{L}(d)=d$, and $\left(i d_{L}(d) \rightarrow 0\right) \odot\left(d \rightarrow i d_{L}(0)\right)=(d \rightarrow 0) \odot(d \rightarrow 0)=d \odot d=c$, we deduce that $i d_{L}(d \rightarrow 0) \neq$ $\left(i d_{L}(d) \rightarrow 0\right) \odot\left(d \rightarrow i d_{L}(0)\right)$, hence $i d_{L}$ is not a pseudo implicative derivation on $L$.

Proposition 3.8. Let $d_{m}$ be a multiplicative derivation on a G-algebra. Then $d_{m}^{2}(x)=d_{m}(x)$, for all $x \in L$.

Proof. Let $x \in L$, since $L$ is a G-algebra, we have $x \odot x=x$ and $d_{m}(x) \odot d_{m}(x)=d_{m}(x)$, then $d_{m}(x)=d_{m}(x \odot x)=d_{m}(x) \odot d_{m}(x) ;$ and we have

$d_{m}^{2}(x)=d_{m}\left(d_{m}(x)\right)=d_{m}\left(d_{m}(x) \odot d_{m}(x)\right)=\left(d_{m}^{2}(x) \odot d_{m}(x)\right) \vee\left(d_{m}(x) \odot d_{m}^{2}(x)\right)=d_{m}^{2}(x) \odot d_{m}(x)$.

Hence $d_{m}^{2}(x) \leq d_{m}(x)$. Now, let's show that $d_{m}(x) \leq d_{m}^{2}(x)$. For that we use the Definition of $d_{m}$ and the fact that $L$ is a G-algebra. We have

$$
\begin{aligned}
\left(d_{m}^{2}(x) \odot x\right) \vee d_{m}(x) & =\left(d_{m}^{2}(x) \odot x\right) \vee\left(d_{m}(x) \odot d_{m}(x)\right)=d_{m}\left(d_{m}(x) \odot x\right) \\
& =d_{m}\left(\left(d_{m}(x) \odot x\right) \vee\left(x \odot d_{m}(x)\right)\right)=d_{m}\left(d_{m}(x \odot x)\right) \\
& =d_{m}\left(d_{m}(x)\right)=d_{m}^{2}(x) .
\end{aligned}
$$

Then, $d_{m}(x) \leq\left(d_{m}^{2}(x) \odot x\right) \vee d_{m}(x)=d_{m}^{2}(x)$. We conclude that $d_{m}^{2}(x)=d_{m}(x)$.

Example 3.9. Let $L$ be the bounded commutative residuated lattice in Example 3.7. Then $L$ is not a G-algebra, we have $d_{m}^{2}(d)=d_{m}\left(d_{m}(d)\right)=0$, hence $d_{m}^{2}(d) \neq d_{m}(d)$.

\section{$4(f, g)$ - multiplicative derivations}

In this section, we extend the notions of a multiplicative derivation by introducing the notion of $(f, g)$-multiplicative derivation and discuss some related properties. The condition for $(f, g)$ multiplicative derivation to be monotone are provided. The set of fixed points is defined where $d$ is $(f, g)$-multiplicative derivation on $L$, and the conditions for the set of fixed points to be a down closed set and an ideal of $L$ are given.

Definition 4.1. Let $L$ be a bounded commutative residuated lattice. A unary operation $d: L \rightarrow L$ is called $(f, g)$-multiplicative derivation on $L$ for the given two homomorphisms $f, g: L \rightarrow L$ if $d(x \odot y)=(d(x) \odot f(y)) \vee(g(x) \odot d(y))$ for all $x, y \in L$.

Remark 4.2. - If we replace the homomorphisms $f$ and $g$ by the identity map, we get a multiplicative derivation. An $(f, f)$-multiplicative derivation is simply called $f$-multiplicative derivation.

- If $d$ is a homomorphism, then $d$ is a $(d, d)$-multiplicative derivation on $L$,

Example 4.3. Let $L$ be the bounded commutative residuated lattice in Example 3.2. We define the functions $f, g$ and $d$ on $L$ by: 


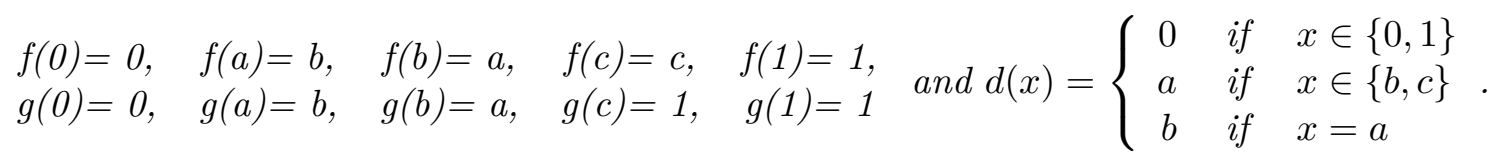

It is easy to verify that $d$ is an $(f, g)$-multiplicative derivation on $L$.

Theorem 4.4. Let $d$ be an $(f, g)$-multiplicative derivation on $L$. Then for all $x, y \in L$, the following conditions hold:

(1) $d(0)=0$,

(2) $d(x) \leq f(x)^{* *}$ and $d(x) \leq g(x)^{* *}$. If $x \leq y$, then $d(x) \leq f(y)^{* *}$ and $d(x) \leq g(y)^{* *}$, and moreover $x \in B(L)$ implies $d(x) \leq f(x)$ and $d(x) \leq g(x)$,

(3) $d(x)=d(x) \vee(d(1) \odot f(x))=d(x) \vee(g(x) \odot d(1))$ and also $f(x) \odot d(1) \odot d(x)^{*}=g(x) \odot$ $d(1) \odot d(x)^{*}=0$,

(4) $d\left(x^{*}\right) \leq d(x)^{*}$,

(5) $d(x)=1$ implies $f(x)^{* *}=1=g(x)^{* *}$,

(6) $d\left(x^{*}\right) \leq f\left(x^{*}\right)$ and $d\left(x^{*}\right) \leq g\left(x^{*}\right)$.

Proof.

(1) Let $d$ be an $(f, g)$-multiplicative derivation on $L$. Then

$$
d(0)=d(0 \odot 0)=(d(0) \odot f(0)) \vee(g(0) \odot d(0))=(d(0) \odot 0) \vee(0 \odot d(0))=0 .
$$

(2) Since $x \odot x^{*}=0$, we have $0=d(0)=d\left(x \odot x^{*}\right)=\left(d(x) \odot f(x)^{*}\right) \vee\left(g(x) \odot d\left(x^{*}\right)\right)$, thus $d(x) \odot f\left(x^{*}\right)=0$. Hence $d(x) \leq f(x)^{* *}$ by Theorem 2.9. By the similar way, we get $d\left(x^{*} \odot x\right)=$ $\left(d\left(x^{*}\right) \odot f(x)\right) \vee\left(g(x)^{*} \odot d(x)\right)=0$, then $g(x)^{*} \odot d(x)=0$, hence $d(x) \leq g(x)^{* *}$.

Always by the similar way, if $x \leq y$, then $x \odot y^{*}=0$, and we get $d(x) \leq f(y)^{* *}$ and $d(x) \leq g(y)^{* *}$. If $x \in B(L)$, then by using $x^{* *}=x$, we conclude that $d(x) \leq f(x)$ and $d(x) \leq g(x)$.

(3) We have,

$$
d(x)=d(x \odot 1)=(d(x) \odot f(1)) \vee(g(x) \odot d(1))=d(x) \vee(g(x) \odot d(1)),
$$

so, $g(x) \odot d(1) \leq d(x)$, hence $g(x) \odot d(1) \odot d(x)^{*}=0$ by Theorem 2.9. We also get $d(x)=$ $d(x) \vee(d(1) \odot f(x))$, thus $f(x) \odot d(1) \leq d(x)$ by Theorem 2.9, hence $f(x) \odot d(1) \odot d(x)^{*}=0$.

(4) We have $d(x) \leq f(x)^{* *}$ by $(2)$, so $d\left(x^{*}\right) \leq f\left(x^{*}\right)^{* *}$ that is $d\left(x^{*}\right) \leq f(x)^{* * *}$. Futhermore $d(0)=d\left(x \odot x^{*}\right)=\left(d(x) \odot f(x)^{*}\right) \vee\left(g(x) \odot d\left(x^{*}\right)\right)=0$; thus, $d(x) \odot f(x)^{*}=f(x)^{*} \odot d(x)$ that is $f(x)^{*} \leq d(x)^{*}$, hence $d\left(x^{*}\right) \leq f(x)^{*}$ and $f(x)^{*} \leq d(x)^{*}$, we conclude that $d\left(x^{*}\right) \leq d(x)^{*}$.

(5) By using (2) and the fact that 1 is the greatest element of $L$, the proof is clear.

(6) Since $x^{* * *}=x^{*}, d(x) \leq f(x)^{* *}$ and $d(x) \leq g(x)^{* *}$, we have $d\left(x^{*}\right) \leq f\left(x^{*}\right)^{* *}=f\left(x^{*}\right)$ and $d\left(x^{*}\right) \leq g\left(x^{*}\right)^{* *}=g\left(x^{*}\right)$, hence $d\left(x^{*}\right) \leq f\left(x^{*}\right)$ and $d\left(x^{*}\right) \leq g\left(x^{*}\right)$.

Theorem 4.5. Let $d$ be an $(f, g)$-multiplicative derivation on $L$ such that $d(1)=1$. Then the following conditions hold:

(1) $f(x) \leq d(x)$ and $g(x) \leq d(x)$, for all $x \in L$, 
(2) $d(B(L))=f(B(L))=g(B(L))$.

Proof. (1) We have $d(x)=d(x) \vee(d(1) \odot f(x))$ and $d(x)=d(x) \vee(g(x) \odot d(1))$ by Theorem 4.4 (3), that is $d(x)=d(x) \vee(1 \odot f(x))=d(x) \vee f(x)$ and $d(x) \vee(g(x) \odot 1)=d(x) \vee g(x)$. Hence $f(x) \leq d(x)$ and $g(x) \leq d(x)$ for all $x \in L$.

(2) $d(x) \leq f(x)$ and $d(x) \leq g(x)$ for all $x \in B(L)$ by Theorem 4.4 (2) and by (1) $f(x) \leq d(x)$ and $g(x) \leq d(x)$, for all $x \in B(L)$. Hence, $g(x)=d(x)=f(x)$ for each $x \in B(L)$ and so $d(B(L))=f(B(L))=g(B(L))$.

Proposition 4.6. Let $d$ be an $(f, g)$-multiplicative derivation on $L$, then we have:

(1) $d(x \odot y) \leq d(x) \vee d(y)$,

(2) $\operatorname{Ker}_{0}(d)=\{x \in L: d(x)=0\}$ is closed under $\odot$.

Proof. (1) It is straightforward to show it.

(2) Let $x, y \in \operatorname{Ker}_{0}(d)$. Then $d(x)=0=d(y)$. By (1), we have $d(x \odot y) \leq d(x) \vee d(y) \leq 0 \vee 0=0$, implying $d(x \odot y) \leq 0$ and so $d(x \odot y)=0$, hence $x \odot y \in \operatorname{Ker}_{0}(d)$.

Proposition 4.7. If $d$ is a homomorphism, then $\operatorname{Ker}_{0}(d)=\{x \in L: d(x)=0\}$ is an ideal.

Proof. - Since $d(0)=0$, we get $0 \in \operatorname{Ker}_{0}(d)$.

- Let $x, y \in L$ such that $x \leq y$ and $y \in \operatorname{Ker}_{0}(d)$. We have $x \leq y$ implies that $d(x) \leq d(y)$, because $d$ is a homomorphism. Then $d(x) \leq d(y)=0$, hence $d(x)=0$.

- Let $x, y \in \operatorname{Ker}_{0}(d)$. Since $d$ is a homomorphism, we have $d(x \rightarrow y)=d(x) \rightarrow d(y)=0 \rightarrow 0$. Hence $\operatorname{Ker}_{0}(d)$ is an ideal of $L$.

Remark 4.8. In the Example 4.马, $d$ is not a homomorphism and $\operatorname{Ker}_{0}(d)$ is not an ideal of $L$.

Definition 4.9. [3] Let $L$ be a lattice, a function $d: L \rightarrow L$ is called generalized $(f, g)$-derivation on $L$ if there exist functions $f, g: L \rightarrow L$ such that $d(x \wedge y)=(d(x) \wedge f(y)) \vee(g(x) \wedge d(y))$, for all $x, y \in L$.

Remark 4.10. If $d$ is an $(f, g)$-multiplicative derivation on the Boolean center $B(L)$, then $d$ is a generalized $(f, g)$ - derivation.

Definition 4.11. Let $d$ be an $(f, g)$-multiplicative derivation on $L$, we denote $F I X_{d}^{f,-}(L)=\{x \in$ $L: d(x)=f(x)\}$ and $F I X_{d}^{-, g}(L)=\{x \in L: d(x)=g(x)\}$.

Example 4.12. In Example 4.3 Fix $x_{d}^{f}(L)=\{0, a, b\}$ and $F i x_{d}^{g}(L)=\{0, a, b\}$.

Theorem 4.13. Let $d$ be an $(f, g)$-multiplicative derivation on the Boolean center $B(L)$. Then the following statements hold:

(1) if $d$ is a homomorphism, then $F I X_{d}^{f,-}(L)$ is an ideal of $L$,

(2) for all $x \in L$, if $x \in F I X_{d}(L)$, then $x^{*} \in F I X_{d}^{f,-}(L)$. 
Proof. (1) Let $x, y \in B(L)$ such that $y \leq x$, and $x \in F I X_{d}^{f,-}(L)$, then $d(x)=f(x)$, and so by Theorem 4.4(2), we have

$$
\begin{aligned}
d(y) & =d(x \wedge y)=d(x \odot y)=(d(x) \odot f(y)) \vee(g(x) \odot d(y)) \\
& =(d(x) \wedge f(y)) \vee(g(x) \wedge d(y))=f(y) \vee d(y)=f(y) .
\end{aligned}
$$

Hence, $y \in F I X_{d}^{f,-}(L)$. Let $x, y \in F I X_{d}^{f,-}(L)$. Then $d(x)=f(x)$ and $d(y)=f(y)$. Since $f$ and $d$ are homomorphisms, we get

$$
f(x \oslash y)=f(x) \oslash f(y)=d(x) \oslash d(y)=d(x \oslash y) .
$$

Hence, $x \oslash y \in F I X_{d}(L)$, we conclude that $F I X_{d}^{f,-}(L)$ is an ideal of $L$.

(2) We have $x \in F I X_{d}^{f,-}(L) \Rightarrow d(x)=f(x)$, so $d(x)^{*}=f(x)^{*}=f\left(x^{*}\right) \leq d\left(x^{*}\right)$, thus $d(x)^{*} \leq$ $d\left(x^{*}\right)$ by Theorem 4.5(1). Futhermore, by Theorem 4.4 (5), $d\left(x^{*}\right) \leq d(x)^{*}$, hence $d\left(x^{*}\right)=$ $d(x)^{*}$, that is $f\left(x^{*}\right)=f(x)^{*}=d\left(x^{*}\right)=d(x)^{*}$, we conclude that $x^{*} \in F I X_{D}^{f}(L)$.

Remark 4.14. - In Example 4.3 $B(L)=\{0,1\}$ and $A=\{a, b, c\}$ is not a Boolean center. We have Fix $_{d}^{f}(L)=\{b, a\}$ is not an ideal of $L$.

- We have for an $(f, g)$-multiplicative derivation d on the Boolean center $B(L)$ :

(3) if $d$ is a homomorphism, then $F I X_{d}^{-, g}(L)$ is an ideal of $L$,

(4) for all $x \in L$, if $x \in F I X_{d}(L)$, then $x^{*} \in F I X_{d}^{-, g}(L)$.

- We have $F I X_{d}^{i d_{L},-}(L)=F I X_{d}^{-, i d_{L}}(L)=F I X_{d}(L)$.

Theorem 4.15. Let $a \in L$, we define a map $d: L \rightarrow L$ by $d(x)=a \odot f(x)$ for all $x \in L$, where $f$ is a homomorphism. Then $d$ is an $f$-multiplicative derivation on $L$.

Proof.

$$
\begin{array}{rlr}
d(x \odot y) & =a \odot f(x \odot y)=a \odot(f(x \odot y) \vee f(x \odot y)) & \\
& =(a \odot f(x \odot y)) \vee(a \odot f(x \odot y)) & \\
& =(a \odot(f(x) \odot f(y)) \vee(a \odot(f(x) \odot f(y)) & \\
& =((a \odot f(x)) \odot f(y)) \vee(f(x) \odot(a \odot f(y))) & \\
& =(d(x) \odot f(y)) \vee(f(x) \odot d(y)) .
\end{array}
$$

It is easy to see that $d$ is a monotone on $L$ (Let $x \leq y$, we have $a \odot x \leq a \odot y$ by Proposition 2.8, thus $d(x) \leq d(y))$.

Proposition 4.16. Let $d: L \rightarrow L$ be a monotone $f$-multiplicative derivation on $L$ such that $d(1) \in B(L)$, then $d(x)=d(1) \odot f(x)$.

Proof. We have $d(x) \leq f(x)$ according to Theorem 4.4, so $d(1) \odot d(x) \leq d(1) \odot f(x)$. Since $d(1) \in B(L)$, we get $d(1) \odot d(x)=d(1) \wedge d(x)=d(x)$ because $x \leq 1$ for all $x \in L$ and $d$ is monotone. So $d(x) \leq d(1) \odot f(x)$. Futhermore, we have $d(1) \odot f(x) \leq d(x) \vee(d(1) \odot f(x))=d(x)$ according to Theorem 4.4. Thus $d(1) \odot f(x) \leq d(x)$. Hence $d(x)=d(1) \odot f(x)$. 


\section{$5(f, g)$ - pseudo implicative derivations}

In the following section, we extend the notion of a pseudo implicative derivation to the notion of $(f, g)$-pseudo implicative derivation and discuss some related properties. The conditions for $(f, g)$ pseudo implicative derivation to be monotone are provided. The condition under which $d(I) \subseteq I$ is given where $d$ is an $(f, g)$-pseudo implicative derivation on $L$ and $I$ is an ideal of $L$.

Definition 5.1. Let $L$ be a bounded commutative residuated lattice. A unary operation $d: L \rightarrow L$ is called $(f, g)$-pseudo implicative derivation on $L$ for the given two homomorphisms $f, g: L \rightarrow L$, if $d(x \rightarrow y)=(d(x) \rightarrow f(y)) \odot(g(x) \rightarrow d(y))$ for all $x, y \in L$.

Remark 5.2. If we replace the homomorphisms $f$ and $g$ by the identity function, we get a pseudo implicative derivation.

An $(f, f)$-pseudo implicative derivation is simply called $f$-pseudo implicative derivation.

If $d$ is a homomorphism on $L$, then $d$ is a $(d, d)$-pseudo implicative derivation on $L$. The statement is also true if $d$ is just a map on $L$ such that $d(x \rightarrow y)=(d(x) \rightarrow d(y))$ for all $x, y \in L$.

Example 5.3. Let $L$ be the bounded commutative residuated lattice in Example 3.2. We define the functions $f, g$ and $d$ on $L$ by:

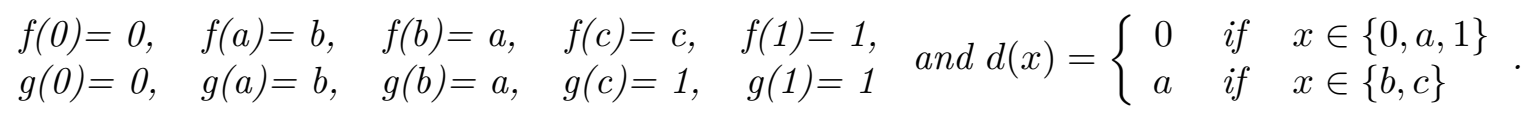

It is easy to verify that $d$ is an $(f, g)$-pseudo implicative derivation on $L$.

Theorem 5.4. Let $d$ be an $(f, g)$-pseudo implicative derivation on $L$, then for all $x, y \in L$, the following statements hold:

(1) $d(0)=0$,

(2) $d(x)=d(x) \odot g(x)$,

(3) $d(x) \leq g(x)$

(4) If $d(x)=1$, then $g(x)=1$,

(5) If $x \in B(L)$, then $d(x) \leq f(x)$,

(6) $d\left(x^{*}\right) \leq(d(x))^{*}$

(7) $d\left(x^{*}\right)=(d(1) \rightarrow f(x)) \rightarrow d(x)$,

(8) If $x \in B(L)$, then $d(x)=(d(1) \odot f(x)) \rightarrow\left(d\left(x^{*}\right)\right)^{*}$,

(9) If $x, y \in B(L)$, then $d(x \wedge y) \leq d(x) \wedge\left(d\left(y^{*}\right)\right)^{*}$,

(10) If $x \leq y$ then $d(x \rightarrow y)=0$.

Proof. (3) and (4) are straightforward.

(1) $d(0)=d(0 \rightarrow x)=(d(0) \rightarrow f(x)) \odot(g(0) \rightarrow d(x))=0$

$(2) d(x)=d(x \rightarrow 0)=(d(x) \rightarrow f(0)) \odot(g(x) \rightarrow d(0))=(d(x) \rightarrow 0) \odot(g(x) \rightarrow d(0))=$ $d(x) \odot g(x)$. 
(5) We have $d(x)=d\left(1 \rightarrow x^{*}\right)=\left(d(1) \rightarrow f\left(x^{*}\right)\right) \odot\left(g(1) \rightarrow d\left(x^{*}\right)\right)=\left(d(1) \rightarrow f\left(x^{*}\right)\right) \odot\left(d\left(x^{*}\right)\right)^{*} \leq$ $d(1) \rightarrow f\left(x^{*}\right) \leq\left(f\left(x^{*}\right)\right)^{*}=f\left(x^{* *}\right)=f(x)$, hence $d(x) \leq f(x)$.

(6) We have $d\left(x^{*}\right)=d(1 \rightarrow x)=(d(1) \rightarrow f(x)) \odot(g(1) \rightarrow d(x))=(d(1) \rightarrow f(x)) \odot(d(x))^{*} \leq$ $(d(x))^{*}$, hence $d\left(x^{*}\right) \leq(d(x))^{*}$.

(7) We have $d\left(x^{*}\right)=d(1 \rightarrow x)=(d(1) \rightarrow f(x)) \odot(g(1) \rightarrow d(x))=(d(1) \rightarrow f(x)) \odot(d(x))^{*}=$ $(d(1) \rightarrow f(x)) \rightarrow d(x)$.

(8) $d(x)=d\left(1 \rightarrow x^{*}\right)=\left(d(1) \rightarrow f\left(x^{*}\right)\right) \odot\left(g(1) \rightarrow d\left(x^{*}\right)\right)=(d(1) \odot f(x)) \odot\left(d\left(x^{*}\right)\right)^{*}$.

(9) $d(x \wedge y)=d(x \odot y)=d\left(x \rightarrow y^{*}\right)=\left(d(x) \rightarrow f\left(y^{*}\right)\right) \odot\left(g(x) \rightarrow d\left(y^{*}\right)\right)=(d(x) \odot f(y)) \odot$ $\left.\left(g(x) \odot\left(d\left(y^{*}\right)\right)^{*}\right)=(d(x) \wedge f(y)) \wedge\left(g(x) \wedge\left(d\left(y^{*}\right)\right)^{*}\right) \leq d(x) \wedge d\left(y^{*}\right)\right)^{*}$.

(10) $d(x \rightarrow y)=d\left(x \odot y^{*}\right)=d(0)=0$.

Proposition 5.5. Let $d$ be an $(f, g)$-pseudo implicative derivation on $L$. Then for all $x \in L$, the following statements hold:

(1) $d(x) \rightarrow g(y) \leq g(x) \rightarrow d(y)$,

(2) $d(x \rightarrow y) \leq d(x) \rightarrow d(y)$.

Proof. (1) By Theorem 5.4 (3) we have $d(x) \leq q(x)$ and $d(y) \leq g(y)$; by using Theorem $2.9\left(L_{19}\right)$ we have $g(y)^{*} \leq d(y)^{*}$ and Proposition $2.8\left(L_{12}\right)$ gives $d(x) \odot(g(y))^{*} \leq g(x) \odot(g(y))^{*} \leq$ $g(x) \odot(d(y))^{*}$. Then $d(x) \rightarrow g(y)=d(x) \odot(g(y))^{*} \leq g(x) \odot(d(y))^{*}=g(x) \rightarrow d(y)$.

$(2) d(x \rightarrow y)=(d(x) \rightarrow f(x)) \odot(g(x) \rightarrow d(y))=\left(d(x) \odot(f(x))^{*}\right) \odot\left(g(x) \odot(d(y))^{*}\right) \leq$ $d(x) \odot(d(y))^{*}=d(x) \rightarrow d(y)$.

Remark 5.6. Let $d$ be an $(f, g)$-pseudo implicative derivation on $L$ and $I$ be an ideal of $L$. If $g(I) \subseteq I$, then $d(I) \subseteq I$.

By using the characterization of ideal given in Theorem 2.6; let $y \in L$ and $y \in d(I)$. We have $y \in d(I)$ implies that there exists $x \in I$ such that $d(x)=y$. By Theorem 5.4(3), we have $d(x) \leq g(x)$, thus $d(x) \odot g\left(x^{*}\right)=0$, futhermore $g(x) \in I$. Hence $y \in I$. The converse is not always true, as we can see in the following example.

Example 5.7. Let $L$ be the bounded commutative residuated lattice in Example 3.7 and d an $(f, g)$ pseudo implicative derivation on $L$ defined as follows:

$$
d(x)=\left\{\begin{array}{lll}
0 & \text { if } & x \in\{0, a, 1\} \\
a & \text { if } & x \in\{b, c\}
\end{array} .\right.
$$

and $I=\{0, a, c\}$ be an ideal on $L$. We have $g(I)=g(\{0, a, c\})=\{0, b, 1\} \nsubseteq I$, and $d(I)=$ $d(\{0, a, c\})=\{0, a\} \subseteq I$.

Theorem 5.8. Let $d$ be an $(f, g)$ - pseudo implicative derivation on L. Then the following statements hold:

(1) $d$ is monotone on $B(L)$, 
(2) If $d(x \rightarrow y)=d(x) \rightarrow d(y)$, then $d$ is monotone on $L$.

Proof. (1) Let $x, y \in B(L)$ such that $x \leq y$. We have $x \leq y \Rightarrow x \wedge y=x$. Futhermore $x \wedge y=x \odot y=x \odot\left(y^{*}\right)^{*}=x \rightarrow y^{*}$. Thus, $d\left(x \rightarrow y^{*}\right)=\left(d(x) \rightarrow f\left(y^{*}\right)\right) \odot\left(g(x) \rightarrow d\left(y^{*}\right)\right) \leq$ $g(x) \rightarrow d\left(y^{*}\right)=g(x) \odot\left(d\left(y^{*}\right)\right)^{*} \leq d\left(y^{* *}\right)=d(y)$.

(2) Let $x, y \in L$ such that $x \leq y$. By Theorem 5.4 (10), we have $d(x \rightarrow y)=0$. Then $0=d(x \rightarrow$ $y)=d(x) \rightarrow d(y)=d(x) \odot(d(y))^{*}$; and we have $d(x) \leq d(y)$.

Proposition 5.9. Let $a \in L$, we define a map $d: L \rightarrow L$ by $d(x)=a \odot f(x)$ for all $x \in L$, where $f$ is a homomorphism. Then $d$ is an $f$-pseudo implicative derivation on $B(L)$.

Proof. We have

$$
\begin{aligned}
(d(x) \rightarrow f(y)) \odot(f(x) \rightarrow d(y)) & =\left(d(x) \odot f(y)^{*}\right) \odot\left(f(x) \odot d(y)^{*}\right) \\
& =(d(x) \odot f(x)) \odot\left(f(y)^{*} \odot d(y)^{*}\right) \\
& =(d(x) \wedge f(x)) \odot\left(f(y)^{*} \wedge d(y)^{*}\right) \\
& =(d(x) \wedge f(x)) \odot(f(y) \vee d(y))^{*} \\
& =d(x) \odot f(y)^{*} \\
& =a \odot f(x) \odot f(y)^{*} \\
& =a \odot f(x \rightarrow y) \\
& =d(x \rightarrow y) .
\end{aligned}
$$

Proposition 5.10. Let $d$ be an $f$-pseudo implicative derivation on $B(L)$. Then for all $x \in L$, $d(x)=d(1) \odot f(x)$.

Proof. We have

$$
\begin{aligned}
d(x)=d\left(1 \rightarrow x^{*}\right) & =\left(d(1) \rightarrow f\left(x^{*}\right)\right) \odot\left(f(1) \rightarrow d\left(x^{*}\right)\right) \\
& =\left(d(1) \odot f\left(x^{*}\right)^{*}\right) \odot d\left(x^{*}\right)^{*} \\
& =\left(d(1) \odot f\left(x^{*}\right)^{*}\right) \odot d\left(x^{*}\right)^{*} \\
& =d(1) \odot\left(f(x) \odot d\left(x^{*}\right)^{*}\right.
\end{aligned}
$$

We have $d(x) \leq f(x)$ that is $d\left(x^{*}\right) \leq f\left(x^{*}\right)$, thus $f\left(x^{*}\right)^{*} \leq d\left(x^{*}\right)^{*}$ that is $f(x) \leq d\left(x^{*}\right)^{*}$, hence $f(x) \wedge d\left(x^{*}\right)^{*}=f(x) \odot d\left(x^{*}\right)^{*}=f(x)$, we conclude that $d(x)=d(1) \odot f(x)$.

\section{$6(f, g)$-implicative derivations}

In this section, we introduce the notion of $(f, g)$-implicative derivations in the bounded commutative residuated lattice and discuss some important properties, we also analyze the link between different types of derivation.

Definition 6.1. Let $L$ be a bounded commutative residuated lattice. A map $d: L \rightarrow L$ is called $(f, g)$-implicative derivation on $L$ for the given two homomorphisms $f, g: L \rightarrow L$, if $d(x \rightarrow y)=$ $(d(x) \rightarrow f(y)) \vee(g(x) \rightarrow d(y))$ for all $x, y \in L$. 
Remark 6.2. If we replace the homomorphisms $f$ and $g$ by the identity map we get an implicative derivation.

An $(f, f)$-implicative derivation is simply called $f$-implicative derivation.

If $d$ is a homomorphism on $L$, then $d$ is a $(d, d)$-implicative derivation on $L$. The statement stay true if $d$ is just a map on $L$ such that $d(x \rightarrow y)=(d(x) \rightarrow d(y))$ for all $x, y \in L$.

Example 6.3. Let $L$ be the bounded commutative residuated lattice in Example 3.2. We define the functions $f, g$ and $d$ on $L$ by:

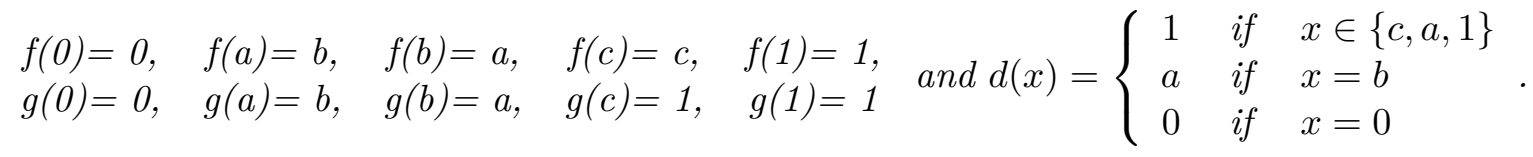

It is easy to verify that $d$ is an $(f, g)$-implicative derivation on $L$.

Theorem 6.4. Let $d$ be an $(f, g)$-implicative derivation, then the following assertions are satisfied:

(1) $d(1)=1$,

(2) $d(x) \leq f(x)$ or $g(x) \leq d(x)$,

(3) If $d(x)=1$ and $g(x) \rightarrow d(x) \neq 1$, then $f(x)=1$,

(4) If $g(x) \rightarrow d\left(x^{* *}\right) \neq 1$, then $d(x) \leq f\left(x^{* *}\right)$,

(5) If $g(x) \rightarrow d(x) \neq 1$ and $g(x) \rightarrow d\left(x^{* *}\right) \neq 1$, then $d\left(x^{*}\right) \leq d(x)^{*}$,

(6) If $g\left(x^{*}\right) \rightarrow d\left(x^{*}\right) \neq 1$, then $d\left(x^{*}\right) \leq f\left(x^{*}\right)$.

Proof.

(1) We have $1=1 \rightarrow 1$, thus $d(1)=d(1 \rightarrow 1)=(d(1) \rightarrow f(1)) \vee(g(1) \rightarrow d(1))=(d(1) \rightarrow 1) \vee(1 \rightarrow$ $d(1))=1 \vee(1 \rightarrow d(1))=1$.

(2) We have $x \rightarrow x=1$, thus $d(1)=(d(x) \rightarrow f(x)) \vee(g(x) \rightarrow d(x))=1$, so $d(x) \rightarrow f(x)=1$ or $g(x) \rightarrow d(x)=1$. We conclude that $d(x) \leq f(x)$ or $g(x) \leq d(x)$.

(3) Since $g(x) \rightarrow d(x) \neq 1$ and $d(x)=1$, thus by (2), we have $d(x) \leq f(x)$ and $1 \leq f(x)$, futhermore 1 is the greatest element of $L$, hence $f(x)=1$.

(4) We have $x \leq x^{* *}$, then $x \rightarrow x^{* *}=1$, thus $d\left(x \rightarrow x^{* *}\right)=\left(d(x) \rightarrow f\left(x^{* *}\right)\right) \vee\left(g(x) \rightarrow d\left(x^{* *}\right)\right)=1$, so $d(x) \rightarrow f\left(x^{* *}\right)=1$, hence $d(x) \leq f(x)^{* *}$.

(5) Since $x^{* * *}=x^{*}$ and $d(x) \leq f(x)^{* *}$, we have $d\left(x^{*}\right) \leq f\left(x^{*}\right)^{* *}=f\left(x^{*}\right)$; futhermore, $d(x) \leq f(x)$, thus $f(x)^{*} \leq d(x)^{*}$ hence $d\left(x^{*}\right) \leq d(x)^{*}$.

(6) $x^{*} \rightarrow x^{*}=1$, then $d\left(x^{*} \rightarrow x^{*}\right)=\left(d\left(x^{*}\right) \rightarrow f\left(x^{*}\right)\right) \vee\left(g\left(x^{*}\right) \rightarrow d\left(x^{*}\right)\right)=1$, thus $d\left(x^{*}\right) \rightarrow f\left(x^{*}\right)=$ 1 , hence $d\left(x^{*}\right) \leq f\left(x^{*}\right)$.

Proposition 6.5. Let $L$ be a bounded commutative residuated lattice and $d: L \rightarrow L$ an $(f, g)$ implicative derivation. Then we have the following statements:

(1) $f(x) \leq d(x)$,

(2) $f(B(L))=d(B(L))$. 
Proof. (1) We have

$$
\begin{aligned}
d(1 \rightarrow x) & =(d(1) \rightarrow f(x)) \vee(g(1) \rightarrow d(x)) \\
& =(1 \rightarrow f(x)) \vee(1 \rightarrow d(x)) \\
& =(1 \rightarrow f(x)) \vee(1 \rightarrow d(x)) \\
& =d(x) \vee f(x))
\end{aligned}
$$

Therefore, $f(x) \leq d(x)$ because $1 \rightarrow x=x$.

(2) We have $f(x) \leq d(x)$ By (1) and by Theorem 6.4(2) we have $d(x) \leq f(x)$, hence $d(x)=f(x)$, we conclude that $f(B(L))=d(B(L))$.

Theorem 6.6. Let $d$ be an $(f, g)$-implicative derivation. We have the following statements:

(1) $d(x) \vee d(y) \leq d(x \rightarrow y)$,

(2) $\operatorname{Ker}(d)=\{x \in L: d(x)=1\}$ is closed for $\rightarrow$.

Proof.

(1) We have $d(x) \leq f(y) \rightarrow d(x)$ and $d(y) \leq g(x) \rightarrow d(y)$ thus

$$
d(x) \vee d(y) \leq(f(y) \rightarrow d(x)) \vee(g(x) \rightarrow d(y))=d(x \rightarrow y) .
$$

(2) Let $x, y \in \operatorname{Ker}(d)$. We have $x, y \in \operatorname{Ker}(d)$ implies that $d(x)=1$ and $d(y)=1$. Since $d(x) \vee d(y) \leq d(x \rightarrow y)$, by (1) we have $1 \vee 1 \leq d(x \rightarrow y)$, that is $1 \leq d(x \rightarrow y)$. Therefore, $d(x \rightarrow y)=1$.

Proposition 6.7. Let $f$ be an endomorphism of a bounded commutative residuated lattice $L$ and $d$ a unary operation defined on $L$ by $d(x)=a \rightarrow f(x)$ for all $x \in L$; where a is a given element of L. Then the following assertions are satisfied:

(1) $d$ is an $f$-implicative derivation on $L$,

(2) d is monotone,

(3) $d(x) \vee d(y) \leq d(x \vee y)$ and $d(x \wedge y)=d(x) \wedge d(y)$, for all $x, y \in L$.

Proof.

(1) Let $f$ be an endomorphism of $L$. Then

$$
\begin{aligned}
(d(x) \rightarrow f(y)) \vee(f(x) \rightarrow d(y)) & =((a \rightarrow f(x)) \rightarrow f(y)) \vee(f(x) \rightarrow(a \rightarrow f(y))) \\
& =((a \rightarrow f(x)) \rightarrow f(y)) \vee(a \rightarrow(f(x) \rightarrow f(y))) \\
& =a \rightarrow(f(x) \rightarrow f(y))=d(x \rightarrow y) .
\end{aligned}
$$

(2) Follows from the fact that $f$ is a homomorphism

(3) Follows from (2), Theorem 2.9 $\left(L_{20}\right)$ and the defintion of $d$.

The following proposition allows us to establish a link between some types of $f$-multiplicative derivation and $f$-implicative derivation. 
Proposition 6.8. Let $L$ be a bounded commutative residuated lattice, $\alpha$ and $\beta$ two $f$-derivations on $L$ defined as follows: $\alpha: L \rightarrow L$ by $\alpha(x)=a \odot f(x)$ and $\beta: L \rightarrow L$ by $\beta(x)=a \rightarrow f(x)$; where $a$ is a given element in $L$. Then $\alpha$ and $\beta$ are monotone; if $\alpha(x) \leq f(y)$, then $f(x) \leq \beta(y)$ and if $f(x) \leq \beta(y)$, then $\alpha(x) \leq f(y)$.

Proof.

(1) By Proposition 2.8 $\left(L_{12}\right)$ and $\left(L_{13}\right) \alpha$ and $\beta$ are monotone.

(2) Since $\alpha(x) \leq f(y)$, we have $a \odot f(x) \leq f(y)$, then $f(x) \leq a \rightarrow f(y)$, and so $f(x) \leq \beta(y)$. Futhermore, since $f(x) \leq \beta(y)$, we get $f(x) \leq a \rightarrow f(y)$, then $a \odot f(x) \leq f(y)$, and so $\alpha(x) \leq$ $f(y)$.

Remark 6.9. If $f$ is the identity map, then $(\alpha, \beta)$ is a Galois connection.

This Proposition highlights the link between the $f$-multiplicative derivation and the $f$-pseudo implicative derivation.

Proposition 6.10. (1) Let $d: L \rightarrow L$ be a monotone $f$-multiplicative derivation on $B(L)$, then $d$ is an $f$-pseudo implicative derivation.

(2) Let d be an $f$-pseudo implicative derivation on $B(L)$, then $d$ is an $f$-multiplicative derivation.

Proof. (1) Follows from Proposition 4.16 and 5.9

(2) Follows from Proposition 5.10 and 4.15

\section{Conclusion}

In this paper, we have studied $(f, g)$ - multilplicative derivation, $(f, g)$-pseudo implicative derivation and $(f, g)$-implicative derivation with clear examples as well as some important properties. As future work, we shall extend the notion of $(f, g)$-derivations in residuated multilattices.

\section{References}

[1] S. Alsatayhi, A. Moussavi, $(\varphi, \psi)$-derivations of BL-algebra, Asian- European Journal of Mathematics, 11(1) (2018), 1-19.

[2] N.O. Alsheri, Derivations of $M V$-algebras, International Journal of Mathematics and Mathematical Sciences, 2010 (2010), 1-7, doi:10.1155/2010/312027.

[3] M. Asci, S. Ceran, $(f, g)$-derivations of lattices, Mathematical Sciences and Applications, 1(2) (2013), 56-62.

[4] R. Bělohlávek, Some properties of residuated lattices, Czechoslovak Mathematical Journal, 53(1) (2003), 161-171.

[5] L. Ferrari, On derivations of lattices, Pure Mathematics and Applications, 12(4) (2001), 365382.

[6] P. He, X. Xinb, J. Zhan, On derivations and their fixed point sets in residuated lattices, Fuzzy Sets and Systems, 303 (2016), 97-113. 
[7] U. Höhle, Commutative, residuated 1-monoids. In: Non-classical logics and their applications to fuzzy subsets, Springer, Dordrecht, 32 (1995), 53-106.

[8] Y. B. Jun, X. L. Xin, On derivations of BCI- algebras, Information Sciences, 159(3-4) (2004), $167-176$.

[9] Y. Lui, Y. Qin, X. Qin, Y. Xu, Ideals and fuzzy ideals on residuated lattice, International Journal of Machine Learning and Cybernetics, 8(1) (2017), 239-253

[10] E.C. Posner, Derivations in prime rings, Proceedings of the American Mathematical Society, 8(6) (1957), 1093-1100.

[11] C. Raluca, J. Antoaneta, On the lattice of congruence filters of a residuated lattice, Annals of the University of Craiova-Mathematics and Computer Science Series, 33 (2006), 174-188.

[12] G. Szász, Derivations of lattices, Acta Scientiarum Mathematicarum, 37(1-2) (1975), 149-154.

[13] M. Ward, R. P. Dilworth, Residuated lattice, Transactions of the American Mathematical Society, 45(3) (1939), 335-354.

[14] Y. Yang, X. Xin, On characterizations of BL-algebras via implicative ideals, Italian Journal of Pure and Applied Mathematics, 37 (2017), 493-506. 